\title{
Some Refined Schwarz-Pick Lemmas
}

\author{
H. TURGAY KAPtANOĞLU
}

\section{Introduction}

There has recently been an unexpected activity in the discovery of some new and sharper versions of the Schwarz lemma (see $[1 ; 2 ; 3 ; 7 ; 8 ; 9]$ ), mostly in the disc. It is our aim in this paper to obtain further forms in the spirit of Pick for functions between discs and between balls of different dimensions.

Several new forms in the disc $[1 ; 7 ; 9]$ consider functions $f$ that map the origin to the origin and then use the functions $f(z) / z$ to refine the estimates given in the classical forms of the Schwarz lemma. We first relax the condition $f(0)=0$ and obtain the Pick versions of these forms. The other generalization comes from replacing the function $f(z) / z$ by a suitable variant for functions between balls. The results in these directions are given in two sections: those pertaining to derivatives in Section 4; and those about the function values in Section 5. In Section 6, we apply the results of Section 4 to obtain quantitative estimates on derivatives at boundary points-in particular, on angular derivatives in discs and in balls. Two earlier sections are for the preliminary material: Section 2 for the more analytic; and Section 3 for the more geometric.

Our notation is fully explained in Section 2, but we mention two points in order to give a sample of our main results. First, $\beta_{n}$ denotes the integrated Bergman metric in the unit ball $\mathbb{B}_{n}$ of $\mathbb{C}^{n}$. Second, $f^{*}(a)=\varphi_{f(a)}^{\prime}(f(a)) f^{\prime}(a) \varphi_{a}^{\prime}(0)$ is the hyperbolic derivative of a holomorphic function $f$ at a point $a \in \mathbb{B}_{n}$, where $\varphi_{a}$ is a Möbius transformation that interchanges 0 and $a$. In this work, the ball case always includes the disc case.

Lemma 4.1. If $f: \mathbb{B}_{n} \rightarrow \mathbb{B}_{m}$ is holomorphic, $f(a)=b$, and $f(A)=B$, then

$$
\beta_{m}\left(S f^{*}(a) \frac{\varphi_{a}(A)}{\left|\varphi_{a}(A)\right|}, T f^{*}(A) \frac{\varphi_{A}(a)}{\left|\varphi_{A}(a)\right|}\right) \leq 2 \beta_{n}(a, A),
$$

where $S$ and $T$ are unitary transformations satisfying $S\left(\varphi_{b}(B)\right)=T\left(\varphi_{B}(b)\right)$.

Corollary 5.3. If $\zeta \in \mathbb{B}_{n}$ then, for all holomorphic $f: \mathbb{B}_{n} \rightarrow \mathbb{B}_{m}$ satisfying $f(a)=b$ and $f(A)=B$, the region of values of $f^{*}(A) \zeta$ is a closed ellipsoid whose center is

Received September 19, 2001. Revision received February 11, 2002.

The author thanks V. E. Katsnelson of the Weizmann Institute for a useful suggestion. 


$$
c_{5}=\frac{1-|P|^{2}}{1-|G(|P| \zeta)|^{2}} \frac{G(|P| \zeta)}{|P|}
$$

and whose radii (see Section 3 ) are

$$
\rho_{5}=\frac{1-|G(|P| \zeta)|^{2} /|P|^{2}}{1-|G(|P| \zeta)|^{2}}|P| \quad \text { and } \quad \sigma_{5}=\sqrt{\frac{1-|G(|P| \zeta)|^{2} /|P|^{2}}{1-|G(|P| \zeta)|^{2}}}|P|,
$$

where $G=\varphi_{B} \circ f \circ \varphi_{A}$ and $P=\varphi_{A}(a)$.

LeMma 6.4. Let $f: \mathbb{B}_{n} \rightarrow \mathbb{B}_{m}$ be holomorphic and satisfy $f(a)=0$. Suppose that $A \in \partial \mathbb{B}_{n}$ and that $f$ has finite angular derivative at $A$. Then

$$
\underset{z \rightarrow A}{\mathrm{r}-\lim _{z \rightarrow}}\left\langle f^{\prime}(z) A, B\right\rangle \geq \frac{1-|a|}{1+|a|},
$$

where $B=\mathrm{r}-\lim _{z \rightarrow A} f(z)$ and $\mathrm{r}$-lim indicates the restricted limit.

\section{Preliminaries}

We use $\mathbb{D}$ for the unit disc in the complex plane $\mathbb{C}$ and $\mathbb{B}_{n}$ for the unit ball in the complex $n$-space $\mathbb{C}^{n}$. Clearly $\mathbb{B}_{1}=\mathbb{D}$. A point in $\mathbb{D}$ or $\mathbb{B}_{n}$ is denoted by the same letter, say $z$, which in the latter case is an $n$-tuple $\left(z_{1}, \ldots, z_{n}\right)$ of complex numbers. The overbar $\left(^{-}\right)$denotes the closure of a set or the complex conjugate of a number, and $\partial$ indicates the topological boundary of a set. For $z \in \mathbb{B}_{n}$, we occasionally put $\zeta_{z}=z /|z| \in \partial \mathbb{B}_{n}$. The inner product and the corresponding norm in $\mathbb{C}^{n}$ are $\langle z, w\rangle=z_{1} \bar{w}_{1}+\cdots+z_{n} \bar{w}_{n}$ and $|z|=\sqrt{\langle z, z\rangle}$. Orthogonality is always with respect to this inner product in this paper.

Our main tools are the slice functions and the automorphisms of balls. If $f$ is a holomorphic function on $\mathbb{B}_{n}$ and $\zeta \in \partial \mathbb{B}_{n}$, then the slice function $f_{\zeta}$ is the restriction of $f$ to the complex line (one-complex-dimensional subspace) $[\zeta]$ through the origin and $\zeta$ and is defined for $\lambda \in \mathbb{D}$ by $f_{\zeta}(\lambda)=f(\lambda \zeta)$. Then $f_{\zeta}$ is holomorphic in $\mathbb{D}$. Given $f: \mathbb{B}_{n} \rightarrow \mathbb{B}_{m}$, the function $f_{\zeta}(\lambda) / \lambda: \mathbb{B}_{n} \rightarrow \mathbb{D}$ is essentially what replaces the quotient $f(z) / z$ for an $f: \mathbb{D} \rightarrow \mathbb{D}$.

The one-to-one holomorphic mappings of $\mathbb{B}_{n}$ onto itself, whose inverses also turn out to be holomorphic, constitute the group of automorphisms of $\mathbb{B}_{n}$. This group is generated by the unitary transformations along with the Möbius transformations $\varphi_{a}(z)$ for $a \in \mathbb{B}_{n}$ given by

$$
\varphi_{a}(z)=\frac{a-X_{a} z-s_{a} Y_{a} z}{1-\langle z, a\rangle},
$$

where $s_{a}=\sqrt{1-|a|^{2}}, X_{a}$ is the projection onto the complex line [a] generated by $a$ and given by

$$
X_{a} z=\frac{\langle z, a\rangle}{|a|^{2}} a
$$

and $Y_{a}=I-X_{a}$. When $n=1$, it follows that $X_{a}=I, Y_{a}=0$, and $\varphi_{a}$ reduces to the familiar 


$$
\varphi_{a}(z)=\frac{a-z}{1-\bar{a} z} .
$$

Each $\varphi_{a}$ is its own inverse and exchanges $a$ and 0 . If $a$ and $A$ are any two points in $\mathbb{B}_{n}$, then $\varphi_{A} \circ \varphi_{a}$ maps $a$ to $A$. The identity

$$
1-\left|\varphi_{a}(A)\right|^{2}=\frac{\left(1-|a|^{2}\right)\left(1-|A|^{2}\right)}{|1-\langle A, a\rangle|^{2}}
$$

is very useful. The explicit formula for $\varphi_{a}$ in the special case $a=\left(a_{1}, 0, \ldots, 0\right)$ is

$$
\varphi_{a}(z)=\left(\frac{a_{1}-z_{1}}{1-\bar{a}_{1} z_{1}}, \frac{-s_{a} z_{2}}{1-\bar{a}_{1} z_{1}}, \ldots, \frac{-s_{a} z_{n}}{1-\bar{a}_{1} z_{1}}\right),
$$

where $s_{a}=\sqrt{1-|a|^{2}}$. Then

$$
\varphi_{a}^{\prime}(0)=-\operatorname{diag}\left\{s_{a}^{2}, s_{a}, \ldots, s_{a}\right\} \quad \text { and } \quad \varphi_{a}^{\prime}(a)=-\operatorname{diag}\left\{\frac{1}{s_{a}^{2}}, \frac{1}{s_{a}}, \ldots, \frac{1}{s_{a}}\right\} .
$$

For much more on the automorphisms of $\mathbb{B}_{n}$, see Chapter 2 of [10] and of [4].

The Möbius transformations give rise to the integrated Bergman metric (the hyperbolic metric, the Poincaré metric in the disc) $\beta$ on $\mathbb{B}_{n}$ that is given by

$$
\beta(z, w)=\frac{1}{2} \log \frac{1+\left|\varphi_{z}(w)\right|}{1-\left|\varphi_{z}(w)\right|}=\tanh ^{-1} \gamma(z, w),
$$

where $\gamma(z, w)=\left|\varphi_{z}(w)\right|$ is the pseudohyperbolic metric. The importance of the Bergman metric lies in the fact that every holomorphic $f: \mathbb{B}_{n} \rightarrow \mathbb{B}_{m}$ is a contraction when the Bergman metric is used for both the domain and the range; that is,

$$
\beta_{m}(f(z), f(w)) \leq \beta_{n}(z, w) .
$$

In particular, the Bergman metric is invariant under the automorphisms of $\mathbb{B}_{n}$ when $m=n$; this statement is true also for the pseudohyperbolic metric.

For an analytic function $f: \mathbb{D} \rightarrow \mathbb{D},[1]$ makes use of the hyperbolic derivative

$$
f^{*}(a)=\frac{1-|a|^{2}}{1-|f(a)|^{2}} f^{\prime}(a)
$$

of $f$ at $a \in \mathbb{D}$, which satisfies $\left|f^{*}(a)\right| \leq 1$ by the Schwarz-Pick lemma. This form is not suitable for use with a function of several complex variables. If $f: \mathbb{B}_{n} \rightarrow \mathbb{B}_{m}$ and $f(a)=b$, we use instead the transformed holomorphic function

$$
g=\varphi_{b} \circ f \circ \varphi_{a}: \mathbb{B}_{n} \rightarrow \mathbb{B}_{m} .
$$

This new function has the properties $f=\varphi_{b} \circ g \circ \varphi_{a}, g(0)=0$, and $g^{\prime}(0)=$ $\varphi_{b}^{\prime}(b) f^{\prime}(a) \varphi_{a}^{\prime}(0)$. The standard form of the Schwarz lemma says that the linear transformation $g^{\prime}(0)$ maps $\mathbb{B}_{n}$ to $\mathbb{B}_{m}$ (see [10, Thm. 8.12]). Following [1], we call $g^{\prime}(0)$ the hyperbolic derivative of $f$ at $a$ and denote it by $f^{*}(a)$. Hence $\left|f^{*}(z) \zeta\right| \leq$ 1 for all $\zeta \in \mathbb{B}_{n}$. In $\mathbb{D}$, no $\zeta$ is required, and equality holds if and only if $f$ is an automorphism of $\mathbb{D}$. When $n=m>1$, a sufficient condition on $f$ to conclude that $f$ is linear is that $f^{\prime}(0)$ be an isometry; see [10, Thm. 8.13]. So if $f^{*}(a)$ is an isometry, then $f$ is an automorphism. 
For the function $f(z)=\varphi_{a}(z)$, [10, Thm. 2.2.2(ii)] immediately gives that $\varphi_{a}^{*}(0)=\varphi_{a}^{*}(a)=-I$, which should be read as -1 when $n=1$. The same method also gives $U^{*}(z)=U^{\prime}(z)=U$ for all $z$ for a unitary $U$. More generally, if $f$ is an automorphism of $\mathbb{B}_{n}$ then so is $g$, and since $g(0)=0$ it follows that $g$ is unitary and thus $f^{*}(z)=g^{\prime}(0)=g=\varphi_{b} \circ f \circ \varphi_{a}$ for any $z \in \mathbb{B}_{n}$.

The hyperbolic derivative is almost invariant under the automorphisms of $\mathbb{B}_{n}$. To make this statement clear, suppose $f: \mathbb{B}_{n} \rightarrow \mathbb{B}_{m}$ is holomorphic and satisfies $f(A)=b$. Let $\psi$ be an automorphism of $\mathbb{B}_{n}$ and put $a=\psi^{-1}(A)$. Now the automorphism $\varphi_{A} \circ \psi \circ \varphi_{a}$ fixes 0 and hence is a unitary transformation $U$, and $\psi \circ \varphi_{a}=\varphi_{A} \circ U$. Then

$$
\begin{aligned}
(f \circ \psi)^{*}(a) & =\varphi_{b}^{\prime}(b)(f \circ \psi)^{\prime}(a) \varphi_{a}^{\prime}(0)=\varphi_{b}^{\prime}(b) f^{\prime}(A) \psi^{\prime}(a) \varphi_{a}^{\prime}(0) \\
& =\varphi_{b}^{\prime}(b) f^{\prime}(A)\left(\psi \circ \varphi_{a}\right)^{\prime}(0)=\varphi_{b}^{\prime}(b) f^{\prime}(A)\left(\varphi_{A} \circ U\right)^{\prime}(0) \\
& =\varphi_{b}^{\prime}(b) f^{\prime}(A) \varphi_{A}^{\prime}(0) U=f^{*}(A) U=f^{*}(\psi(a)) U .
\end{aligned}
$$

Also, if $\zeta \in \mathbb{C}^{n}$ then $\left|(f \circ \psi)^{*}(a) \zeta\right|=\left|f^{*}(\psi(a)) \zeta\right|$.

We list some other properties of the transformed function $g$. We have already indicated that $f(a)=b$ means $g(0)=0$. Similarly, if also $f(0)=0$ then also $g(a)=b$. Now suppose $f$ satisfies only $f(a)=b$ and $f(A)=B$. We can then consider two transformed functions $g=\varphi_{b} \circ f \circ \varphi_{a}$ and $G=\varphi_{B} \circ f \circ \varphi_{A}$. Clearly $g^{\prime}(0)=f^{*}(a)$ and $G^{\prime}(0)=f^{*}(A)$. Now put

$$
p=\varphi_{a}(A), \quad q=\varphi_{b}(B), \quad P=\varphi_{A}(a), \quad \text { and } \quad Q=\varphi_{B}(b) .
$$

Then

$$
q=g(p), \quad Q=G(P), \quad|p|=|P|, \quad \text { and } \quad|q|=|Q| .
$$

This notation is used repeatedly without further mention.

A function $f: \mathbb{B}_{n} \rightarrow \mathbb{B}_{m}$ is said to have finite angular derivative at $A \in \partial \mathbb{B}_{n}$ if there exists a $B \in \partial \mathbb{B}_{m}$ such that

$$
\frac{\langle B-f(z), B\rangle}{\langle A-z, A\rangle}
$$

has finite restricted limit at $A$. We denote the restricted limit by r-lim. (For the definition of the restricted limit we refer to [10, Sec. 8.4.3].) The restricted limit includes the radial limit. On the other hand, we use the notation of [4, Sec. 2.6] and let

$$
d_{f}(A)=\liminf _{z \rightarrow A} \frac{1-|f(z)|}{1-|z|},
$$

where the limit is unrestricted within $\mathbb{B}_{n}$. The Julia-Carathéodory theorem states the equivalence of the following: (i) $d_{f}(A)<\infty$; (ii) $f$ has finite angular derivative at $A$; and (iii) $f$ has restricted limit $B$ at $A$ and $\left\langle f^{\prime}(z) A, B\right\rangle$ has finite restricted limit at $A$. Moreover, when these conditions hold, it states that

$$
\underset{z \rightarrow A}{\mathrm{r}-\lim _{z \rightarrow A}}\left\langle f^{\prime}(z) A, B\right\rangle=d_{f}(A) .
$$

For functions $f: \mathbb{D} \rightarrow \mathbb{D}$, the restricted limit is replaced by the nontangential limit, and we are allowed to use $f^{\prime}(A)$ for the angular derivative at $A$. 


\section{A Little Hyperbolic Geometry}

The balls with center $c$ and radius $r$ in the Bergman, pseudohyperbolic, and Euclidean metrics are denoted by $B_{B}(c, r), B_{P}(c, r)$, and $B(c, r)$, respectively. One general relation between these balls is $B_{B}\left(c, \tanh ^{-1} r\right)=B_{P}(c, r)=\varphi_{c}(B(0, r))$. The automorphism invariance of $\beta$ and $\gamma$ shows that $\varphi_{b}\left(B_{B}(c, r)\right)=B_{B}\left(\varphi_{b}(c), r\right)$ and $\varphi_{b}\left(B_{P}(c, r)\right)=B_{P}\left(\varphi_{b}(c), r\right)$.

When $n=1$ we talk instead about discs denoted by $D$; every $D_{P}$ and hence every $D_{B}$ is also a Euclidean disc whose center and radius are obtained in [6, Sec. I.1]. Thus $D_{B}\left(c, \tanh ^{-1} r\right)=D_{P}(c, r)=\varphi_{c}(D(0, r))=D(C, R)$, where

$$
C=\frac{1-r^{2}}{1-r^{2}|c|^{2}} c \quad \text { and } \quad R=\frac{1-|c|^{2}}{1-r^{2}|c|^{2}} r .
$$

Note that $C \rightarrow 0$ and $c \rightarrow 0$ together, which is forced for example by $r \rightarrow 1$ or $R \rightarrow 1$, which also occur together. Setting $c=0$, we see that $D_{B}\left(0, \tanh ^{-1} r\right)=$ $D_{P}(0, r)=D(0, R)$.

When $n>1$, a non-Euclidean ball is actually a Euclidean ellipsoid as explained in [10, Sec. 2.2.7]. Such an ellipsoid has different radii in different directions: $\rho$ in the complex line $[c]$; and $\sigma$ in all other orthogonal directions. We denote such an ellipsoid by $E(C, \rho, \sigma)$. Thus $B_{B}\left(c, \tanh ^{-1} r\right)=B_{P}(c, r)=\varphi_{c}(B(0, r))=$ $E(C, \rho, \sigma)$, where

$$
C=\frac{1-r^{2}}{1-|c|^{2} r^{2}} c, \quad \rho=\frac{1-|c|^{2}}{1-|c|^{2} r^{2}} r, \quad \text { and } \quad \sigma=\sqrt{\frac{1-|c|^{2}}{1-|c|^{2} r^{2}}} r .
$$

Again $C=0$ and $c=0$ together. When $r$ is small, $\sigma$ can be much larger than $\rho$; but as $r \rightarrow 1$, the difference disappears and both tend to 1 . It is clear from the forms of $\rho$ and $\sigma$ that not every Euclidean ellipsoid is a non-Euclidean ball.

The computation in the reverse direction (i.e., determining the hyperbolic center and radius of a given Euclidean disc situated in $\mathbb{D}$ ) is considerably more involved. It is not easy to find in the literature, so we include it. However, we do not attempt a similar computation in the ball.

Let the disc $D$ given be the Euclidean disc $D(C, R)$, which is also a nonEuclidean disc $D_{B}(c, r)=D_{P}(c, \tanh r)$. We determine $c$ and $r$ in terms of $C$ and $R$. From (7) we know that $C$ and $c$ are in the same direction, so we put $c=t C$ for some $t>1$. Since $D_{B}(c, r)=\varphi_{c}\left(D_{B}(0, r)\right)$ and since the line through 0 and $C$ (or $c$ ) is invariant under $\varphi_{c}$, half the length of the segment $L=[C-R C /|C|$, $C+R C /|C|]$ in the Bergman metric is the hyperbolic radius $r$ of $D$. Therefore,

$$
\gamma\left(C+R \frac{C}{|C|}, C-R \frac{C}{|C|}\right)=\left|\varphi_{C+R C /|C|}\left(C-R \frac{C}{|C|}\right)\right|=\frac{2 R}{1+R^{2}-|C|^{2}}
$$

and

$$
r=\frac{1}{2} \frac{1}{2} \log \frac{1+\frac{2 R}{1+R^{2}-|C|^{2}}}{1-\frac{2 R}{1+R^{2}-|C|^{2}}}=\frac{1}{2} \log \sqrt{\frac{(1+R-|C|)(1+R+|C|)}{(1-R-|C|)(1-R+|C|)}} .
$$

Also, $\tanh r$ is the pseudohyperbolic radius of $D$. 
Now $\varphi_{c}(L)=[(\tanh r) c /|c|,-(\tanh r) c /|c|]$ is another segment symmetric about the origin, so $\varphi_{c}(C-R C /|C|)+\varphi_{c}(C+R C /|C|)=0$. After the substitution $c=t C$ and for $\varphi_{c}$, the last equation eventually leads to the quadratic equation

$$
|C|^{2} t^{2}-\left(1-R^{2}+|C|^{2}\right) t+1=0,
$$

which can be solved for $t$ to obtain

$$
c=\frac{1-R^{2}+|C|^{2}-\sqrt{(1+R-|C|)(1+R+|C|)(1-R-|C|)(1-R+|C|)}}{2|C|^{2}} C .
$$

The minus sign is chosen because, when (7) is substituted in (10), we must have $c=c$. In fact, as a referee pointed out, a plus sign would lead to a $c$ outside $\mathbb{D}$. Likewise, when (7) is substituted in (9), we obtain $r=\tanh ^{-1}(\tanh r)$. Note the similarity between the terms under the radicals in (9) and (10).

\section{Schwarz-Pick Lemmas for Derivatives}

In [1], a classical lemma of Dieudonné is restated and proved in more modern and geometric terms using the Bergman metric. The main result there is that $\beta\left(f^{*}(0), f^{*}(a)\right) \leq 2 \beta(0, a)$ for analytic $f: \mathbb{D} \rightarrow \mathbb{D}$ satisfying $f(0)=0$. This result is also trivially satisfied when $f$ is an automorphism of $\mathbb{D}$, contrary to the implication in [1], because the only origin-preserving automorphisms are rotations and then the left side is 0 . This section concerns the theorem's generalizations to several dimensions and to functions that need not map the origin to the origin.

Lemma 4.1. If $f: \mathbb{B}_{n} \rightarrow \mathbb{B}_{m}$ is holomorphic, $f(a)=b$, and $f(A)=B$, then

$$
\beta_{m}\left(S f^{*}(a) \frac{p}{|p|}, T f^{*}(A) \frac{P}{|P|}\right) \leq 2 \beta_{n}(a, A),
$$

where $S$ and $T$ are unitary transformations satisfying $S(q)=T(Q)$.

Proof. Consider $g, G, p, q, P$, and $Q$. If $\zeta \in \partial \mathbb{B}_{n}$, we pick two unitary rotations $V$ and $W$ on $\mathbb{C}^{n}$ by requiring $V(\zeta)=p /|p|$ and $W(\zeta)=P /|P|$. We pick two other unitary rotations $S$ and $T$ on $\mathbb{C}^{m}$ in such a way that $S(q)=T(Q)$, say, they both equal $(|q|, 0, \ldots, 0)$. And we define two holomorphic functions $h, H: \mathbb{D} \rightarrow \mathbb{B}_{m}$ by

$$
h(\lambda)=\frac{S(g(V(\lambda \zeta)))}{\lambda} \quad \text { and } \quad H(\lambda)=\frac{T(G(W(\lambda \zeta)))}{\lambda} .
$$

Then, using (6), we compute

$$
h(0)=S f^{*}(a) \frac{p}{|p|}, \quad H(0)=T f^{*}(A) \frac{P}{|P|}, \quad \text { and } \quad h(|p|)=H(|P|)=\frac{S(q)}{|p|} \text {. }
$$

Now we apply (4) to $h$ and $H$ and so obtain

$$
\beta(h(0), h(|p|)) \leq \beta(0,|p|) \quad \text { and } \quad \beta(H(0), H(|p|)) \leq \beta(0,|p|)
$$

or (equivalently), by the invariance property of $\beta$,

$$
\beta\left(S f^{*}(a) \frac{p}{|p|}, \frac{S(q)}{|p|}\right) \leq \beta(a, A) \quad \text { and } \quad \beta\left(T f^{*}(A) \frac{P}{|P|}, \frac{S(q)}{|p|}\right) \leq \beta(a, A) \text {. }
$$


In other words, both $S f^{*}(a) p /|p|$ and $T f^{*}(A) P /|P|$ lie in the closed hyperbolic ball $\bar{B}_{B}(S(q) /|p|, \beta(a, A))$. The triangle inequality yields the desired conclusion.

The theorem in [1] is the case $n=m=1$ and $A=B=0$ of Lemma 4.1. Two other less restricted cases are also of interest.

COROllary 4.2. If $f: \mathbb{B}_{n} \rightarrow \mathbb{B}_{m}$ is holomorphic, $f(0)=0$, and $f(a)=b$, then

$$
\beta_{m}\left(f^{*}(0) \frac{a}{|a|}, f^{*}(a) \frac{a}{|a|}\right) \leq 2 \beta_{n}(0, a) .
$$

Proof. We set $A=B=0$ so that $G=f, P=p=a, Q=q=b, T=S$, and $W=V$. Then Lemma 4.1 reads $\beta\left(S f^{*}(0) a /|a|, S f^{*}(a) a /|a|\right) \leq 2 \beta(0, a)$. The invariance of $\beta$ under automorphisms disposes of $S$.

EXAMPLE 4.3. Let's show that the inequality in Corollary 4.2 is sharp. Consider $f\left(z_{1}, \ldots, z_{n}\right)=\left(z_{1}^{2}, 0, \ldots, 0\right)$ and $a=\left(a_{1}, 0, \ldots, 0\right)$. A calculation using $(2)$ and (3) shows that

$$
f^{*}(0)=0 \text { and } f^{*}(a) \frac{a}{|a|}=\left(\frac{2 a_{1}^{2}}{\left(1+\left|a_{1}\right|^{2}\right)\left|a_{1}\right|}, 0, \ldots, 0\right) .
$$

We obtain

$$
\beta_{m}\left(f^{*}(0) \frac{a}{|a|}, f^{*}(a) \frac{a}{|a|}\right)=\frac{1}{2} \log \frac{1+\frac{2\left|a_{1}\right|}{1+\left|a_{1}\right|^{2}}}{1-\frac{2\left|a_{1}\right|}{1+\left|a_{1}\right|^{2}}}=\log \frac{1+\left|a_{1}\right|}{1-\left|a_{1}\right|}=2 \beta_{n}(0, a) .
$$

As a consequence, the function $\varphi_{B} \circ f \circ \varphi_{A}$ gives equality in Lemma 4.1.

The condition in [10, Thm. 8.1.3] that $f^{\prime}(0)$ is an isometry cannot be stated in terms of $f^{*}(0)$ in a simple enough manner. On the other hand, we can easily see that rather different functions give an equality in Corollary 4.2. Let $f\left(z_{1}, \ldots, z_{n}\right)=$ $\left(\left(z_{1}+z_{2}^{2} / 2\right)^{2}, 0, \ldots, 0\right)$ and $a=\left(a_{1}, 0, \ldots, 0\right)$. Another calculation using $(2)$ and (3) shows that $f^{*}(0)$ and $f^{*}(a)$ are as in (11). Hence equality is obtained again in Corollary 4.2. Other examples can be produced from Example 8.1.5(iii) of [10].

Further inequalities can be obtained from Lemma 4.1 and Corollary 4.2. First, if $0 \leq r \leq 1$ then by (4) we have $\beta_{m}\left(r S f^{*}(a) p /|p|, r T f^{*}(A) P /|P|\right) \leq 2 \beta_{n}(a, A)$ and $\beta_{m}\left(r f^{*}(0) a /|a|, r f^{*}(a) a /|a|\right) \leq 2 \beta_{n}(0, a)$. If also $\zeta \in \partial \mathbb{B}_{n}$ and if $f^{*}(a) V=$ $M f^{*}(a)$ and $f^{*}(A) W=N f^{*}(A)$ for some unitary $M$ and $N$ that satisfy $S M=$ $T N$, then $\beta_{m}\left(r f^{*}(a) \zeta, r f^{*}(A) \zeta\right) \leq 2 \beta_{n}(a, A)$ in Lemma 4.1. In the context of Corollary 4.2, this means that $\beta_{m}\left(r f^{*}(0) \zeta, r f^{*}(a) \zeta\right) \leq 2 \beta_{n}(0, a)$ whenever $f^{*}(0) V=M f^{*}(0)$ and $f^{*}(a) V=M f^{*}(a)$. When $n=m=1$, the extra assumptions are not required.

Corollary 4.4. If $f: \mathbb{D} \rightarrow \mathbb{D}$ is analytic, $f(a)=b$, and $f(A)=B$, then

$$
\beta\left(\frac{p}{P} f^{*}(a), \frac{q}{Q} f^{*}(A)\right) \leq 2 \beta(a, A) .
$$


Proof. We pick $n=m=1$ in Lemma 4.1. Then $V$ is a rotation that takes $|p|$ to $p$; so it is multiplication by $p /|p|$. Similarly, $W, S$, and $T$ are multiplications by $P /|P|, \bar{q} /|q|$, and $\bar{Q} /|Q|$, respectively. Using (6) and also the invariance of $\beta$ under automorphisms yields the inequality claimed.

Another inequality that follows from (12) is $\beta\left(r p Q f^{*}(a), r P q f^{*}(A)\right) \leq 2 \beta(a, A)$ for $0 \leq r \leq 1$ by an application of (4).

Equality in the theorem of [1] is obtained precisely for a function of the form $z \psi(z)$, where $\psi$ is an automorphism of $\mathbb{D}$. Hence, equality in (12) is obtained if and only if $f(z)=\varphi_{B}\left(\varphi_{A}(z) \psi\left(\varphi_{A}(z)\right)\right)$. One such function is $f(z)=\varphi_{B}\left(\varphi_{A}(z)^{2}\right)$.

\section{Two-Point Schwarz-Pick Lemmas}

In classical Schwarz-Pick inequalities, the value of $f: \mathbb{D} \rightarrow \mathbb{D}$ at a single point is fixed: at 0 in the Schwarz lemma and at $a \in \mathbb{D}$ in the Schwarz-Pick lemma. Then these lemmas conclude that $f(z)$ lies in the discs $D(0,|z|)$ and $D_{P}(f(a), \rho(z, a))$, respectively. From this point of view, $z$ does not vary and the holomorphic function $f: \mathbb{D} \rightarrow \mathbb{D}$ or $f: \mathbb{B}_{n} \rightarrow \mathbb{B}_{m}$ is the variable entity. Two other classical results, the Dieudonné and Rogosinski lemmas, add more flavor by incorporating also the derivative at $z$ and 0 , respectively (see [5, Sec. 6.3]).

Reference [7] is a rare paper that considers functions that have fixed values at two points $a$ and $A$ and describes the range of values of $f$ at a third point $z$. The surprising point about [7] is that the proof of its main proposition is based on a classical and nonintuitive lemma rather than the simpler and more geometric idea of a non-Euclidean disc. In this section we generalize the results of [7] in several ways by using the more modern and convenient Bergman metric.

Lemma 5.1. Let $z \in \mathbb{B}_{n}$ be fixed. For all holomorphic $f: \mathbb{B}_{n} \rightarrow \mathbb{B}_{m}$ satisfying $f(a)=b$ and $f(A)=B$, the region of values of $f(z)$ is the closed ellipsoid $\bar{E}\left(c_{3}, \rho_{3}, \sigma_{3}\right)$ whose center and radii are described in the proof.

Proof. Consider $G, P$, and $Q$. Let $\zeta \in \partial \mathbb{B}_{n}$ be arbitrary, and define a holomorphic function $H: \mathbb{D} \rightarrow \mathbb{B}_{n}$ by $H(\lambda)=G(\lambda \zeta) / \lambda$. Then $\beta(H(\lambda), H(|P|)) \leq \beta(\lambda,|P|)$ by (4), which means that $H(\lambda)$ lies in the closed hyperbolic ball $\overline{\bar{B}}_{B}(H(|P|)$, $\beta(\lambda,|P|))$. This ball is $\bar{B}_{P}\left(H(|P|),\left|\varphi_{|P|}(\lambda)\right|\right)$ which is the ellipsoid $\bar{E}\left(c_{0}, \rho_{0}, \sigma_{0}\right)$, where (by (8))

$c_{0}=\frac{1-\left|\varphi_{|P|}(\lambda)\right|^{2}}{1-|H(|P|)|^{2}\left|\varphi_{|P|}(\lambda)\right|^{2}} H(|P|), \quad \rho_{0}=\frac{1-|H(|P|)|^{2}}{1-|H(|P|)|^{2}\left|\varphi_{|P|}(\lambda)\right|^{2}}\left|\varphi_{|P|}(\lambda)\right|$,

and $\sigma_{0}$ has a square root around the fraction in $\rho_{0}$. Pick $\lambda=|z|$ and $\zeta=\zeta_{z}=$ $z /|z|$. Then $G(\lambda \zeta)=G(z)=\varphi_{B}\left(f\left(\varphi_{A}(z)\right)\right)$ lies in the ellipsoid $\bar{E}\left(c_{1}, \rho_{1}, \sigma_{1}\right)=$ $\bar{E}\left(|z| c_{0},|z| \rho_{0},|z| \sigma_{0}\right)$.

Now $z$ is fixed but otherwise arbitrary. Hence the preceding argument is valid if $z$ is replaced by $\varphi_{A}(z)$. Thus $\varphi_{B}(f(z))$ lies in the ellipsoid $\bar{E}\left(c_{2}, \rho_{2}, \sigma_{2}\right)$, where 


$$
\begin{aligned}
c_{2} & =\frac{1-\left|\varphi_{|P|}\left(\varphi_{A}(|z|)\right)\right|^{2}}{1-\left|G\left(|P| \zeta_{\varphi_{A}(z)}\right)\right|^{2}\left|\varphi_{|P|}\left(\varphi_{A}(|z|)\right)\right|^{2} /|P|^{2}} \frac{\left|\varphi_{A}(|z|)\right| G\left(|P| \zeta_{\varphi_{A}(z)}\right)}{|P|}, \\
\rho_{2} & =\frac{1-\left|G\left(|P| \zeta_{\varphi_{A}(z)}\right)\right|^{2} /|P|^{2}}{1-\left|G\left(|P| \zeta_{\varphi_{A}(z)}\right)\right|^{2}\left|\varphi_{|P|}\left(\varphi_{A}(|z|)\right)\right|^{2} /|P|^{2}}\left|\varphi_{A}(|z|)\right|\left|\varphi_{|P|}\left(\varphi_{A}(|z|)\right)\right|,
\end{aligned}
$$

and $\sigma_{2}$ has a square root around the fraction in $\rho_{2}$. Note the similarity between $c_{2}$ and $\rho_{2}$, on the one hand, and between $c$ and $r$ of the proposition of [7], on the other. Finally, $f(z)$ lies in the set $C=\varphi_{B}\left(\bar{E}\left(c_{2}, \rho_{2}, \sigma_{2}\right)\right)$. The ellipsoid $E\left(c_{2}, \rho_{2}, \sigma_{2}\right)$ is the image under a Möbius transformation of a ball of some radius centered at the origin. Thus, $C$ is the image of the closure of this ball under some fixed automorphism of $\mathbb{B}_{m}$ and so $C$ is an ellipsoid, which we name $\bar{E}\left(c_{3}, \rho_{3}, \sigma_{3}\right)$.

This lemma does not look very practical as it stands, but several special cases yield interesting results.

Corollary 5.2. Let $z \in \mathbb{B}_{n}$ be fixed. For all holomorphic $f: \mathbb{B}_{n} \rightarrow \mathbb{B}_{m}$ satisfying $f(0)=0$ and $f(a)=b$, the region of values of $f(z)$ is the closed ellipsoid $\bar{E}\left(c_{4}, \rho_{4}, \sigma_{4}\right)$, where

$$
\begin{aligned}
c_{4} & =\frac{1-\left|\varphi_{|a|}(|z|)\right|^{2}}{1-\left|f\left(|a| \zeta_{z}\right)\right|^{2}\left|\varphi_{|a|}(|z|)\right|^{2} /|a|^{2}} \frac{|z| f\left(|a| \zeta_{z}\right)}{|a|}, \\
\rho_{4} & =\frac{1-\left|f\left(|a| \zeta_{z}\right)\right|^{2} /|a|^{2}}{1-\left|f\left(|a| \zeta_{z}\right)\right|^{2}\left|\varphi_{|a|}(|z|)\right|^{2} /|a|^{2}}|z|\left|\varphi_{|a|}(|z|)\right|,
\end{aligned}
$$

and $\sigma_{4}$ has a square root around the fraction in $\rho_{4}$.

Proof. We set $A=B=0$ so that $G=f, P=p=a$, and $Q=q=b$ in Lemma 5.1. To obtain the claimed values, we compute the values of $c_{1}, \rho_{1}$, and $\sigma_{1}$ in this special case.

Corollary 5.3. If $\zeta \in \mathbb{B}_{n}$ then, for all holomorphic $f: \mathbb{B}_{n} \rightarrow \mathbb{B}_{m}$ satisfying $f(a)=b$ and $f(A)=B$, the region of values of $f^{*}(A) \zeta$ is the closed ellipsoid $\bar{E}\left(c_{5}, \rho_{5}, \sigma_{5}\right)$, where

$$
c_{5}=\frac{1-|P|^{2}}{1-|G(|P| \zeta)|^{2}} \frac{G(|P| \zeta)}{|P|}, \quad \rho_{5}=\frac{1-|G(|P| \zeta)|^{2} /|P|^{2}}{1-|G(|P| \zeta)|^{2}}|P|,
$$

and $\sigma_{5}$ has a square root around the fraction in $\rho_{5}$.

Proof. We let $\lambda \rightarrow 0$; then $H(\lambda) \rightarrow G^{\prime}(0) \zeta=f^{*}(A) \zeta$. We next compute the values of $c_{0}, \rho_{0}$, and $\sigma_{0}$ when $\lambda=0$.

Corollary 5.3 is a Dieudonné-Pick lemma for the ball. A Dieudonné lemma for the ball is obtained by further specializing to the case $A=B=0$, in which $G=$ $f$ and $P=a$. This case says that $f^{\prime}(0) \zeta$ lies in the ellipsoid $\bar{E}\left(c_{6}, \rho_{6}, \sigma_{6}\right)$, where

$$
c_{6}=\frac{1-|a|^{2}}{1-|f(|a| \zeta)|^{2}} \frac{f(|a| \zeta)}{|a|}, \quad \rho_{6}=\frac{1-|f(|a| \zeta)|^{2} /|a|^{2}}{1-|f(|a| \zeta)|^{2}}|a|,
$$

and $\sigma_{6}$ has a square root around the fraction in $\rho_{6}$. 
Corollary 5.4. If $z \in \mathbb{B}_{n}$ then, for all holomorphic $f: \mathbb{B}_{n} \rightarrow \mathbb{B}_{m}$ satisfying $f(a)=b$, the region of values of $f(z)$ is the closed ellipsoid $\bar{E}\left(c_{8}, \rho_{8}, \sigma_{8}\right)$ whose center and radii are described in the proof.

Proof. We let $A \rightarrow a$; then $B \rightarrow b, P \rightarrow 0, Q \rightarrow 0$, and $H(|P|) \rightarrow G^{\prime}(0) \zeta=$ $f^{*}(a) \zeta$, where $\zeta=\zeta_{z}$. We next compute the values of $c_{2}, \rho_{2}$, and $\sigma_{2}$ when $P=$ 0 to get $\varphi_{b}(f(z)) \in \bar{E}\left(c_{7}, \rho_{7}, \sigma_{7}\right)$, where

$$
\begin{gathered}
c_{7}=\frac{1-\left|\varphi_{a}(z)\right|^{2}}{1-\left|f^{*}(a) \varphi_{a}(z)\right|^{2}} f^{*}(a) \varphi_{a}(z), \\
\rho_{7}=\frac{1-\left|f^{*}(a) \varphi_{a}(z)\right|^{2} /\left|\varphi_{a}(z)\right|^{2}}{1-\left|f^{*}(a) \varphi_{a}(z)\right|^{2}}\left|\varphi_{a}(z)\right|^{2},
\end{gathered}
$$

and $\sigma_{7}$ has a square root around the fraction in $\rho_{7}$. Hence $f(z)$ lies in

$$
\bar{E}\left(c_{8}, \rho_{8}, \sigma_{8}\right)=\varphi_{b}\left(\bar{E}\left(c_{7}, \rho_{7}, \sigma_{7}\right)\right) .
$$

Corollary 5.4 is a Rogosinski-Pick lemma for the ball. A Rogosinski lemma for the ball is obtained by further specializing to the case $a=b=0$, in which $f^{*}(a)=$ $f^{\prime}(0)$. This case says that $f(z)$ lies in the ellipsoid $\bar{E}\left(c_{9}, \rho_{9}, \sigma_{9}\right)$, where

$$
c_{9}=\frac{1-|z|^{2}}{1-\left|f^{\prime}(0) z\right|^{2}} f^{\prime}(0) z, \quad \rho_{9}=\frac{1-\left|f^{\prime}(0) z\right|^{2} /|z|^{2}}{1-\left|f^{\prime}(0) z\right|^{2}}|z|^{2},
$$

and $\sigma_{9}$ has a square root around the fraction in $\rho_{9}$.

If $f^{*}(a)$ is an isometry in Corollary 5.4, or if $f^{\prime}(0)$ is an isometry in the previous paragraph, then $c_{7}=f^{*}(a) \varphi_{a}(z), c_{9}=f^{\prime}(0) z$, and $\rho_{7}=\sigma_{7}=\rho_{9}=\sigma_{9}=$ 0 as expected by [10, Thm. 8.1.3].

Let's investigate the preceding results for the case $n=m=1$.

Corollary 5.5. Let $z \in \mathbb{D}$ be fixed. For all analytic $f: \mathbb{D} \rightarrow \mathbb{D}$ satisfying $f(a)=b$ and $f(A)=B$, the region of values of $f(z)$ is a closed disc $\bar{D}\left(c_{12}, r_{12}\right)$ whose center and radius are described in the proof.

Proof. We evaluate $c_{2}$ and $\rho_{2}$ of Lemma 5.1 when $n=m=1$, and there is no $\sigma$. Now $H(z)=G(z) / z, \zeta$ does not exist, and we can directly evaluate $H(P)=Q / P$. These lead to the result that $G(z)=\varphi_{B}\left(f\left(\varphi_{A}(z)\right)\right)$ lies in the disc $\bar{D}\left(c_{10}, r_{10}\right)$, where

$$
c_{10}=\frac{1-\left|\varphi_{P}(z)\right|^{2}}{1-|Q / P|^{2}\left|\varphi_{P}(z)\right|^{2}} \frac{z Q}{P} \quad \text { and } \quad r_{10}=\frac{1-|Q / P|^{2}}{1-|Q / P|^{2}\left|\varphi_{P}(z)\right|^{2}}|z|\left|\varphi_{P}(z)\right| .
$$

Then $\varphi_{B}(f(z))$ lies in the disc $\bar{D}\left(c_{11}, r_{11}\right)$, where $c_{11}$ and $r_{11}$ are obtained from $c_{10}$ and $r_{10}$ by replacing $z$ by $\varphi_{A}(z)$. After some manipulation and using (6), these take the forms

$$
c_{11}=\frac{1-\left|\varphi_{a}(z)\right|^{2}}{1-|q / p|^{2}\left|\varphi_{a}(z)\right|^{2}} \frac{\varphi_{A}(z) Q}{P} \text { and } r_{11}=\frac{1-|q / p|^{2}}{1-|q / p|^{2}\left|\varphi_{a}(z)\right|^{2}}\left|\varphi_{A}(z)\right|\left|\varphi_{a}(z)\right| .
$$


Finally, $f(z)$ lies in the disc $\bar{D}\left(c_{12}, r_{12}\right)=\varphi_{B}\left(\bar{D}\left(c_{11}, r_{11}\right)\right)$. The center and radius of this disc can be found as in Section 3.

A simple consequence of Corollary 5.5 is that $|f(z)| \leq\left|c_{12}\right|+r_{12}$. A similar inequality follows also from Corollaries 5.6 and 5.7. These upper bounds for $|f(z)|$ are sharp, as will be shown in Example 5.8.

In Lemma 5.1 and Corollaries 5.2, 5.3, and 5.4, we can likewise conclude that $|f(z)| \leq|c|+\sigma$, where $c$ and $\sigma$ are with appropriate subscripts. But these upper bounds for $|f(z)|$ are clearly not sharp.

The disc case of Corollary 5.2 is the proposition of [7].

Corollary 5.6. For all analytic $f: \mathbb{D} \rightarrow \mathbb{D}$ satisfying $f(a)=b$ and $f(A)=$ $B$, the region of values of $f^{\prime}(A)$ is the closed disc $\bar{D}\left(c_{14}, r_{14}\right)$, where

$$
c_{14}=\frac{1-|P|^{2}}{1-|Q|^{2}} \frac{1-|B|^{2}}{1-|A|^{2}} \frac{Q}{P} \quad \text { and } \quad r_{14}=\frac{|P|^{2}-|Q|^{2}}{|P|\left(1-|Q|^{2}\right)} \frac{1-|B|^{2}}{1-|A|^{2}} .
$$

Proof. Corollary 5.3 tells us that $f^{*}(a)$ lies in the $\operatorname{disc} \bar{D}\left(c_{13}, r_{13}\right)$, where

$$
c_{13}=\frac{1-|P|^{2}}{1-|Q|^{2}} \frac{Q}{P} \quad \text { and } \quad r_{13}=\frac{|P|^{2}-|Q|^{2}}{|P|\left(1-|Q|^{2}\right)} .
$$

Employing (7) finishes the proof.

This is a Dieudonné-Pick lemma. The case $A=B=0$, in which also $P=a$ and $Q=b$, is a Dieudonné lemma and is in [7].

COROLlaRy 5.7. For all analytic $f: \mathbb{D} \rightarrow \mathbb{D}$ satisfying $f(a)=b$, the region of values of $f(z)$ is the closed disc $\bar{D}\left(c_{16}, r_{16}\right)$ whose center and radius are described in the proof.

Proof. Corollary 5.4 tells us that $\varphi_{b}(f(z))$ lies in the disc $\bar{D}\left(c_{15}, r_{15}\right)$, where

$$
c_{15}=\frac{1-\left|\varphi_{a}(z)\right|^{2}}{1-\left|\varphi_{a}(z)\right|^{2}\left|f^{*}(a)\right|^{2}} \varphi_{a}(z) f^{*}(a)
$$

and

$$
r_{15}=\frac{1-\left|f^{*}(a)\right|^{2}}{1-\left|\varphi_{a}(z)\right|^{2}\left|f^{*}(a)\right|^{2}}\left|\varphi_{a}(z)\right|^{2} .
$$

Hence $f(z)$ lies in the $\operatorname{disc} \bar{D}\left(c_{16}, r_{16}\right)=\varphi_{b}\left(\bar{D}\left(c_{15}, r_{15}\right)\right)$.

This is a Rogosinski-Pick lemma. The case $a=b=0$ is a Rogosinski lemma and can also be found in [7].

It is shown in [7] that the functions $G(z)=z \varphi_{Q / P}\left(\alpha \varphi_{P}(z)\right)$ cover the disc $D\left(c_{10}, r_{10}\right)$ as $\alpha$ varies in $\mathbb{D}$. Then the functions $f(z)=\varphi_{B}\left(G\left(\varphi_{A}(z)\right)\right)$ cover the disc $D\left(c_{12}, r_{12}\right)$ as $\alpha$ varies in $\mathbb{D}$. We can extend these examples to several variables, at least when $n>1$ and $m=1$. 
ExAmple 5.8. Let $a=\left(a_{1}, 0, \ldots, 0\right)$ with $a_{1}>0$ for simplicity, $b \in \mathbb{D}, \alpha \in$ $\mathbb{D}$, and $f(z)=z_{1} \varphi_{b / a_{1}}\left(\alpha \varphi_{a}(z)_{1}\right)$, where we invoke (2). For any $\alpha \in \mathbb{D}$, we have $f(0)=0$ and $f(a)=b$. Now look at only $z=\left(z_{1}, 0, \ldots, 0\right)$ with $z_{1}>0$. Then $\zeta_{z}=(1,0, \ldots, 0)$ and $f\left(|a| \zeta_{z}\right)=b$. With also $\mu=\left(a_{1}-z_{1}\right) /\left(1-a_{1} z_{1}\right)$, we obtain

$$
c_{4}=\frac{1-\mu^{2}}{1-|b|^{2} \mu^{2} / a_{1}^{2}} \frac{z_{1} b}{a_{1}} \quad \text { and } \quad r_{4}=\rho_{4}=\frac{1-|b|^{2} / a_{1}^{2}}{1-|b|^{2} \mu^{2} / a_{1}^{2}} z_{1}|\mu| .
$$

We compute to see

$$
\frac{f(z)-c_{4}}{r_{4}}=\frac{\mu}{|\mu|} \varphi_{b \mu / a_{1}}(\alpha),
$$

which covers $\mathbb{D}$ as $\alpha$ varies in $\mathbb{D}$. Thus the functions $f(z)$ cover the disc $D\left(c_{4}, r_{4}\right)$ as $\alpha$ varies in $\mathbb{D}$. We can take care of the other cases of Corollary 5.2 when $m=$ 1 by using unitary rotations; to deal with Lemma 5.1 when $m=1$, we resort to functions of the form $\varphi_{B} \circ f \circ \varphi_{A}$.

\section{Boundary Derivatives}

A development in a different direction is the boundary Schwarz lemma of [9], where a lower bound for the derivative at a boundary point is found. The result is derived from an interior Schwarz lemma, which is nothing but a Rogosinski lemma and which can be found in [5] with the same proof (just add the moduli of the two fractions on top of p. 201) and in [7] with a different proof (just perform $\left.\left|c_{2}\right|+r_{2}\right)$. In this section, we present Pick forms in the disc and in the ball. We base our proofs on the results in Sections 3 and 5 to highlight the geometric nature of the problem.

Lemma 6.1. Let $f: \mathbb{D} \rightarrow \mathbb{D}$ be analytic and satisfy $f(a)=b$. Suppose $A, B \in$ $\partial \mathbb{D}$ and that $f$ has a radial limit $B$ and radial derivative $f^{\prime}(A)$ at $A$. Then

$$
\left|f^{\prime}(A)\right| \geq \frac{2}{1+\left|f^{*}(a)\right|} \frac{1-|b|}{1+|b|} \frac{1-|a|}{1+|a|} .
$$

Proof. By Corollary 5.7, $\varphi_{b}(f(z))$ lies in the disc $\bar{D}\left(c_{15}, r_{15}\right)$, which is (say) $\bar{D}_{P}\left(c_{17}, r_{17}\right)$. Then $f(z)$ lies in the disc $\varphi_{b}\left(\bar{D}_{P}\left(c_{17}, r_{17}\right)=\bar{D}_{P}\left(\varphi_{b}\left(c_{17}\right), r_{17}\right)\right.$, whose Euclidean center and radius are called $c_{16}$ and $r_{16}$ in Corollary 5.7. By (7),

$$
c_{16}=\frac{1-r_{17}^{2}}{1-r_{17}^{2}\left|\varphi_{b}\left(c_{17}\right)\right|^{2}} \varphi_{b}\left(c_{17}\right) \quad \text { and } \quad r_{16}=\frac{1-\left|\varphi_{b}\left(c_{17}\right)\right|^{2}}{1-r_{17}^{2}\left|\varphi_{b}\left(c_{17}\right)\right|^{2}} r_{17} \text {. }
$$

It follows that

$$
|f(z)| \leq\left|c_{16}\right|+r_{16}=\frac{r_{17}+\left|\varphi_{b}\left(c_{17}\right)\right|}{1+r_{17}\left|\varphi_{b}\left(c_{17}\right)\right|}
$$

and

$$
\frac{1-|f(z)|}{1-|z|} \geq \frac{1-\left(\left|c_{17}\right|+r_{17}\right)}{1-|z|}=\frac{1-r_{17}}{1-|z|} \frac{1-\left|\varphi_{b}\left(c_{17}\right)\right|}{1+r_{17}\left|\varphi_{b}\left(c_{17}\right)\right|} .
$$


As $|z| \rightarrow 1$, we have $\left|\varphi_{a}(z)\right| \rightarrow 1, c_{15} \rightarrow 0$, and $r_{15} \rightarrow 1$. Then, by (7), also $c_{17} \rightarrow 0$ and $r_{17} \rightarrow 1$. Therefore,

$$
\liminf _{z \rightarrow A} \frac{1-|f(z)|}{1-|z|} \geq \frac{1-|b|}{1+|b|} \lim _{|z| \rightarrow 1} \frac{1-r_{17}}{1-|z|} .
$$

It remains to compute the limit in (13).

Observe that $r_{17}$ can be obtained by substituting $c_{15}$ and $r_{15}$ for $C$ and $R$ in (9). This substitution, after some tedious work and obvious simplifications, gives

$$
r_{17}=\tanh \left(\log \sqrt{\frac{\sqrt{\omega}}{1-\left|\varphi_{a}(z)\right|^{2}}}\right)
$$

where $\omega=\left(1+\left|\varphi_{a}(z)\right|^{2}\right)^{2}-4\left|\varphi_{a}(z)\right|^{2}\left|f^{*}(a)\right|^{2}$. Using the definition of tanh, $r_{17}$ takes the form

Then, by (1),

$$
r_{17}=\frac{\sqrt{\omega}-\left(1-\left|\varphi_{a}(z)\right|^{2}\right)}{\sqrt{\omega}+\left(1-\left|\varphi_{a}(z)\right|^{2}\right)}
$$

$$
\begin{aligned}
\frac{1-r_{17}}{1-|z|} & =\frac{2\left(1-|a|^{2}\right)(1+|z|)}{|1-\bar{a} z|^{2}\left(\sqrt{\omega}+1-\left|\varphi_{a}(z)\right|^{2}\right)} \\
& \geq \frac{(1-|a|)(1+|z|)}{1+|a|} \frac{2}{\sqrt{\omega}+1-\left|\varphi_{a}(z)\right|^{2}} .
\end{aligned}
$$

Thus, leaving the case $\left|f^{*}(a)\right|=1$ aside,

$$
\lim _{|z| \rightarrow 1} \frac{1-r_{17}}{1-|z|} \geq \frac{1-|a|}{1+|a|} \frac{2}{\sqrt{1-\left|f^{*}(a)\right|^{2}}} \geq \frac{1-|a|}{1+|a|} \frac{2}{1+\left|f^{*}(a)\right|} .
$$

Finally, under the hypotheses of the lemma, $z=|z| A$ and

$$
\left|f^{\prime}(A)\right|=\left|\lim _{|z| \rightarrow 1} \frac{B-f(z)}{A-|z| A}\right| \geq \liminf _{z \rightarrow A} \frac{1-|f(z)|}{1-|z|} .
$$

This completes the proof.

As a consequence, since $\left|f^{*}(a)\right| \leq 1$, we also have

$$
\left|f^{\prime}(A)\right| \geq \frac{1-|b|}{1+|b|} \frac{1-|a|}{1+|a|} .
$$

EXAMPLE 6.2. Looking back at the statement of Lemma 6.1, equality holds in (14) if and only if $\left|f^{*}(a)\right|=1$, which holds (as mentioned in Section 2) if and only if $f$ is an automorphism of $\mathbb{D}$, satisfying of course $f(a)=b$. In fact, let $f(z)=$ $\varphi_{b}\left(e^{i \theta} \varphi_{a}(z)\right)$, where $e^{i \theta}=-\bar{a} b /|a b|$. Then

$$
\left|f^{\prime}(z)\right|=\frac{1-|b|^{2}}{\left|1-\bar{b} e^{i \theta} \varphi_{a}(z)\right|^{2}} \frac{1-|a|^{2}}{|1-\bar{a} z|^{2}} .
$$

Let $A=-a /|a|$ so that $\varphi_{a}(A)=-A=a /|a|$. Substituting $A$ for $z$, using the particular value of $\theta$ chosen, and simplifying, we obtain equality in (14). 
In particular, the hypotheses of Lemma 6.1 are satisfied (by the Julia-Carathéodory theorem) when $f$ has finite angular derivative $f^{\prime}(A)$ at $A$. Then Lemma 6.1 and what follows its proof give lower bounds for the modulus of the angular derivative of $f$ at $A$ and for the $d_{f}(A)$ mentioned in Section 2 .

REMARK 6.3. We can say something also about the region of values of $f^{\prime}(A)$ in Lemma 6.1, at least when $a=b=0$. Corollary 5.7 in this case (actually a Rogosinski lemma) says that $f(z)$ lies in the disc $\bar{D}\left(c_{18}, r_{18}\right)$, where

$$
c_{18}=\frac{1-|z|^{2}}{1-|z|^{2}\left|f^{\prime}(0)\right|^{2}} z f^{\prime}(0) \quad \text { and } \quad r_{18}=\frac{1-\left|f^{\prime}(0)\right|^{2}}{1-|z|^{2}\left|f^{\prime}(0)\right|^{2}}|z|^{2}
$$

are obtained from $c_{15}$ and $r_{15}$ by setting $a=0$. Then the difference quotient $(B-f(z)) /(A-z)$ lies in the disc $\bar{D}\left(c_{19}, r_{19}\right)$, where

$$
c_{19}=\frac{B-c_{18}}{A-z} \quad \text { and } \quad r_{19}=\frac{r_{18}}{|A-z|} .
$$

Now, as $z$ approaches $A$ radially, we have $z=|z| A$ and $|z| \rightarrow 1$, and both $c_{19}$ and $r_{19}$ tend to infinity. However, the term $-c_{18}$ in the numerator of $c_{19}$ is bounded, and $c_{19}$ is asymptotic to $\bar{A} B /(1-|z|)$. Similarly, $r_{19}$ is asymptotic to $1 /(1-|z|)$ as $z \rightarrow A$ radially. These discs enlarge to fill the half-plane $J=\{w: \operatorname{Re}(A \bar{B} w) \geq 0\}$ as $z \rightarrow A$ radially. Thus $f^{\prime}(A)$ lies in the intersection of $J$ and the complement of the disc $D\left(0,2 /\left(1+\left|f^{\prime}(0)\right|\right)\right)$.

Proving a result in the ball in the generality of Lemma 6.1 would require either a computation of derivatives of automorphisms at arbitrary points or a computation of $\rho_{8}$ of Corollary 5.4 in a way similar to that in Section 3. Either computation is prohibitively complicated, so we are content with the following simpler form.

Lemma 6.4. Let $f: \mathbb{B}_{n} \rightarrow \mathbb{B}_{m}$ be holomorphic and satisfy $f(a)=0$. Suppose that $A \in \partial \mathbb{B}_{n}$ and that $f$ has finite angular derivative at $A$. Then

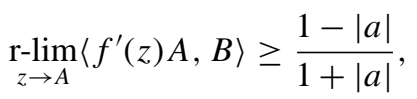

where $B=\mathrm{r}-\lim _{z \rightarrow A} f(z)$.

Proof. By the case $b=0$ of Corollary 5.4, $f(z)$ lies in the ellipsoid $\bar{E}\left(c_{7}, \rho_{7}, \sigma_{7}\right)$. It is impossible to find an upper bound on $|f(z)|$ as directly as in the proof of Lemma 6.1. However, the intersection of $\bar{E}\left(c_{7}, \rho_{7}, \sigma_{7}\right)$ with $\left[c_{7}\right]$ and with its orthogonal complement has circular symmetry. Hence the distance between the origin and the points of the planar ellipse

$$
\frac{\left(x-\left|c_{7}\right|\right)^{2}}{\rho_{7}^{2}}+\frac{y^{2}}{\sigma_{7}^{2}}=1
$$

farthest from the origin is also the same as the distance between the origin and the points of $\bar{E}\left(c_{7}, \rho_{7}, \sigma_{7}\right)$ farthest from the origin. We therefore first solve the Lagrange multiplier problem of finding the maximum of the function $F(x, y)=$ $\sqrt{x^{2}+y^{2}}$ subject to condition (15); we obtain the maximum as 


$$
K=\sqrt{\frac{\sigma^{2}\left(\left|c_{7}\right|^{2}+\sigma^{2}-\rho^{2}\right)}{\sigma^{2}-\rho^{2}}} .
$$

It follows that $|f(z)| \leq K$. Substituting the values of $c_{7}, \sigma_{7}$, and $\rho_{7}$ into the expression for $K$ yields $K=\left|\varphi_{a}(z)\right|$. Thus,

$$
\begin{aligned}
\frac{1-|f(z)|}{1-|z|} & \geq \frac{1-K}{1-|z|}=\frac{1-\left|\varphi_{a}(z)\right|^{2}}{(1-|z|)\left(1+\left|\varphi_{a}(z)\right|\right)} \\
& =\frac{\left(1-|a|^{2}\right)\left(1-|z|^{2}\right)}{|1-\langle a, z\rangle|^{2}(1-|z|)\left(1+\left|\varphi_{a}(z)\right|\right)} \geq \frac{1-|a|}{1+|a|} \frac{1+|z|}{1+\left|\varphi_{a}(z)\right|}
\end{aligned}
$$

and

$$
d_{f}(A)=\liminf _{z \rightarrow A} \frac{1-|f(z)|}{1-|z|} \geq \frac{1-|a|}{1+|a|} .
$$

The proof is complete by the remarks on angular derivatives and the Julia-Carathéodory theorem in Section 2.

EXAMPLE 6.5. We modify Example 6.2 and obtain a function that shows that equality occurs in Lemma 6.4. Let $m=n, f(z)=\varphi_{a}(z)$, and $A=-a /|a|$. This function is holomorphic in a neighborhood of $\bar{B}_{n}$, so we have $B=f(A)=-A$ and $f^{\prime}(A)$ exists. Imitating the proof of [10, Thm. 2.2.2(ii)], we obtain

$$
f^{\prime}(A)=-\frac{1-|a|}{1+|a|} X_{a}-\sqrt{\frac{1-|a|}{1+|a|}} Y_{a}
$$

Since $X_{a}(A)=A$ and $Y_{a}(A)=0$,

immediately follows.

$$
\left\langle f^{\prime}(A) A, B\right\rangle=\frac{1-|a|}{1+|a|}
$$

\section{References}

[1] A. F. Beardon, The Schwarz-Pick lemma for derivatives, Proc. Amer. Math. Soc. 125 (1997), 3255-3256.

[2] A. F. Beardon and T. K. Carne, A strengthening of the Schwarz-Pick inequality, Amer. Math. Monthly 99 (1992), 216-217.

[3] D. M. Burns and S. G. Krantz, Rigidity of holomorphic mappings and a new Schwarz lemma at the boundary, J. Amer. Math. Soc. 7 (1994), 661-676.

[4] C. C. Cowen and B. D. MacCluer, Composition operators on spaces of analytic functions, Stud. Adv. Math., CRC Press, Boca Raton, FL, 1995.

[5] P. L. Duren, Univalent functions, Grundlehren Math. Wiss., 259, Springer, New York, 1983.

[6] J. B. Garnett, Bounded analytic functions, Pure Appl. Math., 96, Academic Press, New York, 1981.

[7] P. R. Mercer, Sharpened versions of the Schwarz lemma, J. Math. Anal. Appl. 205 (1997), 509-511.

[8] R. Osserman, A new variant of the Schwarz-Pick-Ahlfors lemma, Manuscripta Math. 100 (1999), 123-129. 
[9] - A sharp Schwarz inequality on the boundary, Proc. Amer. Math. Soc. 128 (2000), 3513-3517.

[10] W. Rudin, Function theory in the unit ball of $\mathbb{C}^{n}$, Grundlehren Math. Wiss., 241, Springer, New York, 1980.

Mathematics Department

Middle East Technical University

Ankara 06531

Turkey

kaptan@math.metu.edu.tr 